\title{
Dynamic Changes of Peripheral Blood Markers Predict Prognosis of Patients with Advanced Non- Small Cell Lung Cancer Receiving Anti-PD-1/PD-L1 Treatment
}

\author{
Gaoyan Tang \\ Weifang People's Hospital \\ Yan Liu \\ Weifang People's Hospital \\ Qingyun Zhang \\ Weifang People's Hospital \\ Baohong Yang \\ Weifang People's Hospital \\ Yanhong Ding ( $\sim$ yhding19861018@163.com ) \\ Weifang People's Hospital \\ Guohua Yu \\ Weifang People's Hospital
}

\section{Research Article}

Keywords: NLR, PLR, aNSCLC, Immunotherapy, Prognosis

Posted Date: January 21st, 2022

DOI: https://doi.org/10.21203/rs.3.rs-1248240/v1

License: (a) (i) This work is licensed under a Creative Commons Attribution 4.0 International License.

Read Full License 


\section{Abstract}

Background Immune checkpoint inhibitors have been routinely used in the treatment of advanced nonsmall-cell lung cancer (aNSCLC) with significantly improved survival rate. However, the identification of reliable prognositic and predictive biomarkers is lacking. The aim of the study was to investigate the prognostic value of neutrophil-to-lymphocyte ratio (NLR) and platelet-to-lymphocyte ratio (PLR) and their dynamic changes for aNSCLC.

Methods We retrospectively analyzed 60 patients with aNSCLC who received immunotherapy. The NLR and PLR were assessed at baseline (NLRO and PLRO) and after 4 cycles of treatment (NLR4C and PLR4C).

Results Of all aNSCLC patients on immunotherapy, 47 (78.3\%) cases were in the response group. Whether at baseline or after 4 cycles of treatment, the levels of NLR and PLR in the response group were both significantly lower than those in the non-response group $(p<0.05)$. Patients with a high NLR4c had poor progression-free survival (PFS) and overall survival (OS) independently in multivariate analysis (3.90 vs. 7.50 months, $p=0.005 ; 7.20$ vs. 15.90 months, $p=0.041$, respectively). Patients with high NLR0highNLR4c were associated with poor PFS and OS ( $p=0.009, p=0.001$ respectively). Results are similar for PLR.

Conclutions NLR after 4 cycles of treatment and dynamic changes of NLR and PLR during immunotherapy might help to predict a more accurate prognosis in aNSCLC patients.

\section{Introduction}

Immunotherapy has recently made a breakthrough in patients with advanced non-small cell lung cancer (aNSCLC). Immune checkpoint inhibitors (ICls) targeting the programmed cell death 1(PD-

1)/programmed cell death 1 ligand (PD-L1) axis have revolutionized cancer treatment and provided a new option for the treatment of aNSCLC 2. At present, the anti-PD-1 antibodies nivolumab and pembrolizumab have shown superiority with regards to overall response rates (ORR), overall survival (OS) and progression free survival (PFS) compared to chemotherapy for aNSCLC patients 3-5. However, the effective rate of ICls alone in the unscreened NSCLC population is usually less than $20 \% 6$, and $9 \%-29 \%$ patients experience rapid progression of the disease after immunotherapy 7 . It has been shown that the OS of NSCLC patients who failed to respond to nivolumab is 1.4 months, while the patients who respond to treatment can reach 13.5 months 8 . Besides, Immunotherapy is expensive and increases the social economic burden. Therefore, it is important to predict the therapeutic effect of ICls, screen out patients who can benefit from immunotherapy 9 , and maximize the efficacy of immunotherapy for the precise treatment of aNSCLC.

Neutrophil-lymphocyte ratio (NLR) and platelet-lymphocyte ratio (PLR) can reflect the inflammatory level of the body 10 . Several studies have shown that NLR and PLR are associated with patient prognosis in multiple solid tumors, such as gastric cancer 11, colorectal cancer 12, and NSCLC 
13. And some studies have reported that preoperative detection of NLR and PLR levels has some predictive effect on postoperative recurrence in patients with early-stage NSCLC 14,15. However, Reports on the predictive value of NLR, PLR and especially their dynamic changes for the response of immunotherapy in patients with advanced NSCLC are researched rarely. In this study, we retrospectively analyzed the prognostic role of peripheral blood NLR and PLR in 60 patients with aNSCLC in terms of immune efficacy, progression-free survival (PFS) and overall survival (OS). Additionally, we also assessed the dynamics of NLR and PLR during immunotherapy.

\section{Results}

\section{Clinical characteristics of patients}

A total of 60 patients with aNSCLC treated with immunotherapy were included in this study. Baseline characteristics of patients can be seen in Table 1. Patients' median age was 63 (ranging from 30 to 77 years), with a higher proportion of current/former smokers (71.7\%). ECOG was either 0 or 1 for $55(91.7 \%)$ patients, 2 for $5(8.3 \%)$ patients. 18 patients (30.0\%) achieved partial response, 29 (48.3\%) patients experienced stable disease, and $13(21.7 \%)$ patients had progressive disease.

Table 1 Patients' characteristics at baseline and treatment response 


\begin{tabular}{|c|c|c|}
\hline Characteristics & No.of patients $(n=60)$ & Percentage (\%) \\
\hline \multicolumn{3}{|l|}{ Gender } \\
\hline Male & 47 & 78.3 \\
\hline Female & 13 & 21.7 \\
\hline \multicolumn{3}{|l|}{ Age (years) } \\
\hline$\leq 65$ & 35 & 58.3 \\
\hline$>65$ & 25 & 41.7 \\
\hline \multicolumn{3}{|l|}{ Smoking } \\
\hline Never & 17 & 28.3 \\
\hline Former/current & 43 & 71.7 \\
\hline \multicolumn{3}{|l|}{ ECOG } \\
\hline $0-1$ & 55 & 91.7 \\
\hline 2 & 5 & 8.3 \\
\hline \multicolumn{3}{|l|}{ Actionable mutation } \\
\hline$(-) /$ undetected & 43 & 71.7 \\
\hline EGFR or ALK/ROS1(+) & 17 & 28.3 \\
\hline \multicolumn{3}{|l|}{ Histology } \\
\hline Adenocarcinoma & 39 & 65.0 \\
\hline Squamous cell carcinoma & 21 & 35.0 \\
\hline \multicolumn{3}{|l|}{ No. of metastasis sites } \\
\hline 0 & 2 & 3.3 \\
\hline 1 & 22 & 36.7 \\
\hline 2 & 15 & 25.0 \\
\hline$\geq 3$ & 21 & 35.0 \\
\hline \multicolumn{3}{|c|}{ Immunotherapy applied in which line } \\
\hline 1 & 20 & 33.3 \\
\hline 2 & 14 & 23.3 \\
\hline$\geq 3$ & 26 & 43.4 \\
\hline
\end{tabular}




\begin{tabular}{|c|c|c|}
\hline$<3$ & 22 & 36.7 \\
\hline$\geq 3$ & 38 & 63.3 \\
\hline \multicolumn{3}{|l|}{ NLR4c } \\
\hline$<3$ & 38 & 63.3 \\
\hline$\geq 3$ & 22 & 36.7 \\
\hline \multicolumn{3}{|l|}{ PLRO } \\
\hline$<180$ & 38 & 63.3 \\
\hline$\geq 180$ & 22 & 36.7 \\
\hline \multicolumn{3}{|l|}{ PLR4c } \\
\hline$<180$ & 45 & 75.0 \\
\hline$\geq 180$ & 15 & 25.0 \\
\hline \multicolumn{3}{|l|}{ Change in NLR } \\
\hline LowNLR0-LowNLR4c & 16 & 26.7 \\
\hline LowNLR0-HighNLR4c & 6 & 10.0 \\
\hline HighNLR0-LowNLR4c & 22 & 36.6 \\
\hline HighNLR0-HighNLR4c & 16 & 26.7 \\
\hline \multicolumn{3}{|l|}{ Change in PLR } \\
\hline LowPLR0-LowPLR4c & 30 & 50.0 \\
\hline LowPLR0-HighPLR4c & 8 & 13.3 \\
\hline HighPLR0-LowPLR4c & 15 & 25.0 \\
\hline HighPLR0-HighPLR4c & 7 & 11.7 \\
\hline \multicolumn{3}{|l|}{ Best response } \\
\hline CR & 0 & 0.0 \\
\hline PR & 18 & 30.0 \\
\hline SD & 29 & 48.3 \\
\hline PD & 13 & 21.7 \\
\hline
\end{tabular}

ECOG, Eastern Cooperative Oncology Group; NLR0, neutrophil-to-lymphocyte ratio at baseline; NLR4c, neutrophil-to- lymphocyte ratio after 4 cycles of treatment; PLRO, Platelet-to-lymphocyte ratio at baseline; PLR4c, Platelet-to-lymphocyte ratio after 4 cycles of treatment; CR, Complete response; PR, Partial response; SD, Stable disease; PD, Progressive disease. 


\section{Comparison of NLR and PLR between the response and non-response group}

Of all aNSCLC patients on immunotherapy, 47 (78.3\%) cases were in the response group (18 cases PR, 29 $\mathrm{SD})$. The levels of NLRO in the response group and the non-response group were $3.9(2.5,4.6)$ and 5.2 $(4.1,5.8)$, respectively $(p=0.016)$ and PLRO in these two groups were $153.8(96.9,192.2)$ and 229.2 $(166.5,270.5)$, respectively $(p=0.048)$. Besides, the levels of NLR4c and PLR4c in the response group were both significantly lower than those in the non-response group [NLR4c, $2.5(1.5,2.8)$ vs $6.2(4.1,7.0)$, $p<0.001$. PLR4c, $124.2(66.2,159.8)$ vs 250.1 (145.0, 333.9), $p=0.002$ ] respectively (Fig. 1).

\section{NLR, PLR and other factors for PFS}

We conducted a univariate analysis of NLR, PLR and other factors, as shown in Table 2. Age, NLR0, NLR4c, PLR0 and PLR4c were associated with PFS (Table 2, Fig.2). But in multivariate analysis, only NLR4c $<3$ was significantly associated with longer PFS (hazard ratio [HR]: $0.452,95 \%$ Cl: 0.261-0.784, $p=$ 0.005), while PLR0 had marginal significance $(p=0.052)$.

Table 2 Univariate and Multivariate Analysis for PFS 


\section{Variable}

Univariate analysis PFS(months) $\quad 95 \% \mathrm{Cl}$ $p$ value

Multivariate analysis

$\mathrm{HR} \quad 95 \% \mathrm{Cl}$ $p$ value

\begin{tabular}{|c|c|c|c|}
\hline \multicolumn{2}{|l|}{ Gender } & \multirow{2}{*}{$\begin{array}{l}0.134 \\
6.864-7.936\end{array}$} & \\
\hline Male & 7.40 & & \\
\hline Female & 6.90 & $3.462-10.338$ & \\
\hline Age (yrs) & & 0.047 & 0.078 \\
\hline$\leq 65$ & 7.40 & $6.820-7.980$ & \\
\hline$>65$ & 6.90 & 6.778-7.022 & \\
\hline Smoking & & 0.426 & \\
\hline Never & 7.20 & $6.528-7.872$ & \\
\hline Former/current & 7.30 & $6.586-8.014$ & \\
\hline ECOG & & 0.147 & \\
\hline $0-1$ & 7.20 & 6.764-7.636 & \\
\hline 2 & 7.40 & $0.000-17.915$ & \\
\hline Actionable mutation & & 0.987 & \\
\hline$(-) /$ undetected & 7.40 & 7.017-7.783 & \\
\hline $\begin{array}{l}\text { EGFR or } \\
\text { ALK/ROS1(+) }\end{array}$ & 6.90 & 6.303-7.497 & \\
\hline Histology & & 0.728 & \\
\hline Adenocarcinoma & 7.40 & 6.951-7.849 & \\
\hline $\begin{array}{l}\text { Squamous cell } \\
\text { carcinoma }\end{array}$ & 6.90 & $6.222-7.578$ & \\
\hline $\begin{array}{l}\text { No. of metastasis } \\
\text { sites }\end{array}$ & & 0.859 & \\
\hline 0 & 7.90 & & \\
\hline 1 & 7.10 & 6.443-7.757 & \\
\hline 2 & 5.00 & $1.213-8.787$ & \\
\hline$\geq 3$ & 7.40 & 6.733-8.067 & \\
\hline $\begin{array}{l}\text { Immunotherapy } \\
\text { applied in }\end{array}$ & & 0.333 & \\
\hline
\end{tabular}




\section{which line}

\begin{tabular}{lllll}
\hline 1 & 7.40 & $6.743-8.057$ & & \\
\hline 2 & 7.20 & $5.367-9.033$ & & 0.149 \\
\hline$\geq 3$ & 6.90 & $0.315-6.283$ & & \\
\hline NLR0 & & 0.079 & & 0.005 \\
\hline$<3$ & 7.90 & $7.649-8.121$ & 0.452 & \\
\hline$\geq 3$ & 6.80 & $6.199-7.401$ & 1 & 0.784 \\
\hline NLR4C & & 0.003 & & \\
\hline$<3$ & 7.50 & $7.097-7.903$ & & 0.135 \\
\hline$\geq 3$ & 3.90 & $3.674-4.126$ & & \\
\hline PLR0 & & 0.012 & & \\
\hline$<180$ & 7.60 & $7.117-8.083$ & & \\
\hline$\geq 180$ & 6.40 & $4.929-7.871$ & & \\
\hline PLR4c & & 0.013 & & \\
\hline$<180$ & 7.40 & $7.138-7.662$ & & \\
\hline$\geq 180$ & 5.00 & $2.633-7.367$ & & \\
\hline
\end{tabular}

\section{NLR, PLR and other factors for OS}

The OS was estimated using the Kaplan-Meier method, and the results were compared across groups using the log-rank test. Patients' age, NLR4c and PLRO were found to be significantly associated with OS (Table 3, Fig.3). However, in multivariate analysis, age $\leq 65$ and NLR4c $<3$ were significant prognostic factors for OS (HR: $0.310,95 \%$ Cl: 0.168-0.573, p < 0.001, HR: 0.393, 95\% Cl: 0.170-0.542, $p<0.001$, respectively).

Table 3 Univariate and Multivariate Analysis for OS 
Variable

\begin{tabular}{|c|c|c|c|c|}
\hline & & & $\begin{array}{l}\mathrm{HR} \\
\quad p \text { value }\end{array}$ & $95 \% \mathrm{Cl}$ \\
\hline Gender & & 0.235 & & \\
\hline Male & 15.30 & $14.404-16.196$ & & \\
\hline Female & 13.60 & $5.967-21.233$ & & \\
\hline Age (yrs) & & $<0.001$ & $0.168-0.573$ & $<0.001$ \\
\hline$\leq 65$ & 15.90 & 14.393-17.407 & 0.310 & \\
\hline$>65$ & 12.80 & $12.310-13.290$ & 1 & \\
\hline Smoking & & 0.916 & & \\
\hline Never & 14.30 & $12.821-15.779$ & & \\
\hline Former/current & 14.90 & $13.101-16.699$ & & \\
\hline ECOG & & 0.140 & & \\
\hline $0-1$ & 14.90 & $13.656-16.144$ & & \\
\hline 2 & 28.40 & $0.000-74.562$ & & \\
\hline Actionable mutation & & 0.978 & & \\
\hline$(-) /$ undetected & 15.10 & $12.659-17.541$ & & \\
\hline EGFR or ALK/ROS1(+) & 14.80 & $13.859-15.741$ & & \\
\hline Histology & & 0.919 & & \\
\hline Adenocarcinoma & 15.10 & $12.558-17.642$ & & \\
\hline $\begin{array}{l}\text { Squamous cell } \\
\text { carcinoma }\end{array}$ & 14.80 & $13.087-16.513$ & & \\
\hline No. of metastasis sites & & 0.985 & & \\
\hline 0 & 15.30 & - & & \\
\hline 1 & 14.00 & $11.357-16.643$ & & \\
\hline 2 & 11.70 & $1.349-22.051$ & & \\
\hline$\geq 3$ & 15.10 & $14.053-16.147$ & & \\
\hline $\begin{array}{l}\text { Immunotherapy applied in } \\
\text { which line }\end{array}$ & & 0.364 & & \\
\hline 1 & 15.60 & $13.847-17.353$ & & \\
\hline
\end{tabular}


NLRO

0.544

0.363

$<3$

14.30

11.887-16.713

$\geq 3$

14.90

13.392-16.408

NLR4C

$<0.001$

$0.170-0.542$

$<0.001$

$<3$

15.90

14.450-17.350

0.393

$\geq 3$

7.20

6.281-8.119

1

PLRO

0.034

0.086

$<180$

15.60

14.996-16.204

$\geq 180$

11.70

6.874-16.526

PLR4C

0.065

0.521

$<180$

15.50

14.514-16.486

$\geq 180$

12.60

4.016-21.184

\section{Relationship of dynamic changes of NLR and PLR with immune efficacy}

Statistical analysis showed that $100 \%$ (37/37) of patients with low NLRO-low NLR4c and $100 \%(5 / 5)$ of patients with high NLRO-low NLR4c were both in the response group. However, the proportion of patients with high NLR0-high NLR4c and low NLR0-high NLR4C in the non-response group were $61.5 \%(8 / 13)$ and $100 \%(5 / 5)$, respectively (supplementary table S1). After analysis, the immune efficacy of patients with low NLRO-low NLR4c and high NLR0-low NLR4c was significantly better than that of patients with high NLRO-high NLR4c and low NLR0-high NLR4c $(p<0.001)$, and the relationship between PLR dynamic changes and immune efficacy was similar to that of NLR dynamic changes $(p<0.001)$.

\section{Relationship of dynamic changes of NLR and PLR with survival outcomes}

We examined the effects of NLR and PLR changes after initial treatments, wherein the PFS and OS were analyzed according to the change in NLR or PLR. Kaplan-Meier analysis revealed that patients with low 
NLR0-low NLR4c showed a significantly longer PFS and OS. Compared with a median PFS of 7.90 months for patients with low NLR0-low NLR4c, patients with high NLRO-high NLR4c had the worst PFS (median PFS 3.80 months) ( $p=0.009)$ (Fig.4a). Analyses according to PLR showed similar results (Fig.4b). In addition, patients with high NLR0-high NLR4c had the shortest survival $(p=0.001)$ and patients with high PLR0 -high PLR4c also had the shortest survival (although $p=0.075$ ) (Fig. 4c and Fig.4d).

\section{Discussion}

Immunotherapy has provided substantial benefit in NSCLC with unprecedented results in terms of overall in both first- and second-line therapies. Screen patients who benefit from immunotherapy accurately and thereby achieve more accurate individualized immunotherapy. So it is significant to find biomarkers for predicting the efficacy of immunotherapy.

Immune microenvironment in cancer patients is very complex, and the inflammatory response of the body is important for the occurrence and development of tumors 15. The immune microenvironment in which tumor cells are located infiltrates a large number of inflammatory cells, including neutrophils, lymphocytes, platelets, and natural killer cells. Neutrophils, as the main inflammatory cells, can secrete vascular endothelial growth factor, which promotes the generation of tumor blood vessels. Besides, neutrophils can inhibit activated $\mathrm{T}$ lymphocytes, and further weaken the anti-tumor immune response. Many studies have found that neutrophils are closely related to the occurrence and development of tumors in multiple systems of the human body, such as renal cancer, gastric cancer, esophageal cancer, and lung cancer 16-19. Lymphocytes, especially tumor-infiltrating lymphocytes (TILs), play a central role in specific tumor immune responses. TILs are critical in forming the immune environment which is considered to be the "seventh major labeling feature" of tumors 20 . As the main exerciser of cellular immunity, lymphocytes play a major anti-tumor immune effect in cancer patients 21 . Platelets can produce platelet-derived endothelial growth factor and transforming growth factor- $\beta$, which contribute to the growth of tumor cells, and platelets in circulating blood can form polymers with circulating tumor cells (CTCs), allowing CTCs to achieve immune escape and ultimately leading to tumor metastasis 22. Thus, having high NLR and PLR, with a high neutrophil or platelet count and/or low lymphocyte count can contribute to poor prognosis in a variety of cancers.

NLR is considered to be an important indicator of prognosis in many diseases, including the prognosis of chronic obstructive pulmonary disease and silicosis 23-25. Meanwhile, NLR and PLR have also been used as a predictor of malignant tumor prognosis 26-28. Diem et al. found in patients with metastatic NSCLC treated with nivolumab, elevated pretreatment NLR and PLR were associated with shorter OS and PFS as well as reduced response rates, regardless of other prognostic factors 29. Our study found high PLR at baseline was also an independent prognostic factor for PFS and OS in univariate analysis, but NLR at baseline was not significantly associated with prognosis, which was consisted with previous reports 30,31 . Besides, we found that both NLR before and after 4 cycles of treatment were related to the efficacy of immunotherapy $(p<0.05)$, but the relationship between NLR after 4 cycles of treatment and efficacy 
was more significant $(p<0.001)$. The relationship between PLR and efficacy was similar to that of NLR. Further, we demonstrated that the NLR after 4 cycles of treatment better reflect the prognosis than the NLR at baseline, however, PLR at baseline seemed to be more closely related to PFS and OS than posttreatment PLR. In our study on multivariable analysis, only NLR after 4 cycles of treatment was strongly associated with survival and could be used as a more accurate predictor for prognosis.

NLR and PLR are conveniently accessible in the clinic, display high reproducibility, and their dynamic changes are easy to observe. Dynamic changes of NLR and PLR are related to the survival of patients with tumors 32-34. In our study, we analyzed the dynamic changes of NLR and PLR during immunotherapy and found patients with low NLR at baseline and low NLR after 4 cycles of treatment (low NLR0-low NLR4c) had the best immune efficacy and NSCLC patients with high NLR0-low NLR4c had similar efficacy to the former. However, patients with high NLRO-high NLR4c had poor immune effect. The immune efficacy of NSCLC patients with low NLRO-low NLR4c was better than that of patients with high NLR0-high NLR4c. The prediction of immune efficacy by dynamic changes of PLR is similar to the dynamic changes of NLR. Therefore, the dynamic changes of NLR and PLR before and after treatment are related to the efficacy of immunotherapy.

In summary, we found that repeated mesaurements of NLR and PLR have greater value in assessing treatment efficacy. We choose at baseline and after 4 cycles of treatment as the time point and found that NLR after 4 cycles of treatment was a more accurate prognostic predictor. Systemic inflammation represented by NLR and PLR predicts the PFS and OS of patients with advanced NSCLC who are receiving immunotherapy. In addition, dynamic changes of NLR and PLR during immunotherapy might be useful to predict post-treatment prognosis in advanced NSCLC and tailor the therapy after.

Our study has several limitations. First, NLR and PLR can reflect the inflammatory changes of immune microenvironment, but the mechanism of their prediction of efficacy and prognosis is still unclear. At present, the most prominent problem in many related clinical studies is that there is no uniform standard for the cut-off values of NLR and PLR, and a large sample of prospective clinical studies are needed for more in-depth exploration in the future. Second, this study is a single-center, small-sample clinical study, which may have influence on the conclusion, so studies with large sample size are still needed to further explore its relationship with the prognosis. Anyway, the detection of NLR and PLR is simple, cheap and has high patient compliance. their changes before and after immunotherapy in patients with advanced small cell lung cancer are related to the therapeutic efficacy, which may be a predictor of disease control and a biomarker to predict the prognosis. It has the value of further exploration and research and is worthy of being widely popularized in clinical practice.

\section{Methods}

\section{Ethical approval of study}

A retrospective, single-center cohort study was conducted in accordance with the guidelines for good clinical practice (GCP) and the declaration of Helsinki for experiments involving humans. The study was 
approved by the ethical committee of Weifang Medical University, Weifang, China (reference number 2020YX034) and all patients had signed the informed consent.

\section{Participants and clinical treatment}

Sixty patients with aNSCLC who underwent immunotherapy in the Department of Oncology, Weifang People's Hospital from January 2018 to May 2019 were selected. The inclusion criteria were as follows: age over 18 years; patients with histologically or cytologically diagnosed NSCLC; clinical stage IIIB or IV; The Eastern Cooperative Oncology Group (ECOG) Performance Status (PS) score 0-2. Exclusion criteria: patients with acute cardiac disease or chronic underlying diseases, which may interfere with the treatment after evaluation; patients with severe liver and kidney function damage; patients in the terminal stage, who cannot tolerate immunotherapy; patients with autoimmune system diseases. The clinical data of patients were collected and recorded, including age, gender, clinical stage, pathological type, EGFR gene mutation detection, etc. Nivolumab was administered at a dose of $3 \mathrm{mg} / \mathrm{kg}$ i.v. every 14 days. Pembrolizumab was administered at a dose of $2 \mathrm{mg} / \mathrm{kg}$ i.v. every 21 days. Sintilimab and Tislelizumab were administered at a dose of $200 \mathrm{mg}$ i.v. every 21 days and Camrelizumab was administered at a dose of $200 \mathrm{mg}$ i.v. every 14 days.

\section{Evaluation}

Radiological imaging performed before the start of ICls and during treatment was reviewed. The efficacy was evaluated according to the WHO evaluation criteria for solid tumors 1.1 (RECIST1.1). The evaluation includes complete response (CR), partial response (PR), stable disease (SD) and progressive disease (PD). Patients with CR or PR or SD were defined as clinical response, while patients with PD were defined as non-clinical response.

\section{Hematological parameters}

The neutrophils count, lymphocytes count and platelets count at baseline and after 4 cycles of treatment were collected, respectively. The values of NLR and PLR were calculated as follows: NLR = peripheral blood neutrophil count/lymphocyte count, PLR = peripheral blood platelet count/lymphocyte count. The prespecified cutoff values were NLR $\geq 3$ (high NLR) versus $<3$ (low NLR) 13 and PLR $\geq 180$ (high PLR) versus $<180$ (low PLR) 35.

\section{Survival times}

PFS was defined as the time from anti-PD-1/PD-L1 antibody initiation to disease progression or death due any cause, and OS was defined as the time from anti-PD-1/PD-L1 antibody initiation to the last follow-up or death, whichever came first.

\section{Statistical analysis}


SPSS 26.0 statistical software was used for data analysis. Normally distributed measurement data were expressed as Mean \pm SD using $t$ test and measurement data with non-normal distribution were expressed by $\mathrm{Md}(\mathrm{P} 25, \mathrm{P} 75)$ using two independent sample rank sum test. Enumeration data were expressed as percentages using the chi-square test. In addition, the OS and PFS were assessed and compared using the Kaplan-Meier method and the log-rank test. Multivariate analysis for PFS and OS were performed using the variables that were significant on univariate analysis. $p<0.05$ was considered statistically significant.

\section{Declarations}

\section{Data Availability statement}

The data used to support the findings of this study are available from the corresponding author upon request.

\section{Author contributions}

GYT and GHY conceived the idea, developed the theory and interpreted the results. GYT drafted the manuscript. YL and QYZ carried out the data collection. BHY conducted the statistical analysis. YHD revised the manuscript. All authors discussed the results and contributed to the final manuscript.

\section{Acknowledgements}

This work was supported by the Shandong Medical and Health Technology Development Project (Grant No. 2019WS248)

\section{Conflict of interest}

The authors declare that they have no conflict of interest.

\section{References}

1. Gridelli, C. et al. Second-line Treatment of Advanced Non-small Cell Lung Cancer Non-oncogene Addicted: New Treatment Algorithm in the Era of Novel Immunotherapy. Curr Clin Pharmacol 13, 7684, doi:10.2174/1574884713666180711160008 (2018).

2. Russo, A. et al. The changing scenario of 1 (st) line therapy in non-oncogene addicted NSCLCs in the era of immunotherapy. Critical reviews in oncology/hematology 130, 1-12, doi:10.1016/j.critrevonc.2018.06.007 (2018).

3. Brahmer, J. et al. Nivolumab versus Docetaxel in Advanced Squamous-Cell Non-Small-Cell Lung Cancer. The New England journal of medicine 373, 123-135, doi:10.1056/NEJMoa1504627 (2015).

4. Borghaei, H. et al. Nivolumab versus Docetaxel in Advanced Nonsquamous Non-Small-Cell Lung Cancer. The New England journal of medicine 373, 1627-1639, doi:10.1056/NEJMoa1507643 
(2015).

5. Herbst, R. S. et al. Pembrolizumab versus docetaxel for previously treated, PD-L1-positive, advanced non-small-cell lung cancer (KEYNOTE-010): a randomised controlled trial. Lancet (London, England) 387, 1540-1550, doi:10.1016/s0140-6736(15)01281-7 (2016).

6. Garon, E. B. et al. Pembrolizumab for the treatment of non-small-cell lung cancer. The New England journal of medicine 372, 2018-2028, doi:10.1056/NEJMoa1501824 (2015).

7. Ferrara, R. et al. Hyperprogressive Disease in Patients With Advanced Non-Small Cell Lung Cancer Treated With PD-1/PD-L1 Inhibitors or With Single-Agent Chemotherapy. JAMA oncology 4, 15431552, doi:10.1001/jamaoncol.2018.3676 (2018).

8. Costantini, A. et al. Nivolumab-refractory patients with advanced non-small-cell lung cancer. Lung cancer (Amsterdam, Netherlands) 130, 128-134, doi:10.1016/j.lungcan.2019.01.015 (2019).

9. Gibney, G. T., Weiner, L. M. \& Atkins, M. B. Predictive biomarkers for checkpoint inhibitor-based immunotherapy. The Lancet. Oncology 17, e542-e551, doi:10.1016/s1470-2045(16)30406-5 (2016).

10. Lee, S. et al. Prognostic significance of neutrophil lymphocyte ratio and platelet lymphocyte ratio in advanced gastric cancer patients treated with FOLFOX chemotherapy. BMC cancer 13, 350, doi:10.1186/1471-2407-13-350 (2013).

11. Miyamoto, R. et al. The neutrophil-to-lymphocyte ratio (NLR) predicts short-term and long-term outcomes in gastric cancer patients. European journal of surgical oncology: the journal of the European Society of Surgical Oncology and the British Association of Surgical Oncology 44, 607612, doi:10.1016/j.ejso.2018.02.003 (2018).

12. Zhang, J., Zhang, H. Y., Li, J., Shao, X. Y. \& Zhang, C. X. The elevated NLR, PLR and PLT may predict the prognosis of patients with colorectal cancer: a systematic review and meta-analysis. Oncotarget 8, 68837-68846, doi:10.18632/oncotarget.18575 (2017).

13. Nakaya, A. et al. Neutrophil-to-lymphocyte ratio as an early marker of outcomes in patients with advanced non-small-cell lung cancer treated with nivolumab. International journal of clinical oncology 23, 634-640, doi:10.1007/s10147-018-1250-2 (2018).

14. Pinato, D. J. et al. Prognostic performance of inflammation-based prognostic indices in primary operable non-small cell lung cancer. British journal of cancer 110, 1930-1935, doi:10.1038/bjc.2014.145 (2014).

15. Zhang, H. et al. Clinical significance of preoperative neutrophil-lymphocyte vs platelet-lymphocyte ratio in primary operable patients with non-small cell lung cancer. American journal of surgery 210, 526-535, doi:10.1016/j.amjsurg.2015.03.022 (2015).

16. Byun, S. S. et al. Prognostic Significance of Preoperative Neutrophil-to-Lymphocyte Ratio in Nonmetastatic Renal Cell Carcinoma: A Large, Multicenter Cohort Analysis. BioMed research international 2016, 5634148, doi:10.1155/2016/5634148 (2016).

17. Wang, S. C. et al. Pretreatment Neutrophil to Lymphocyte Ratio Independently Predicts Diseasespecific Survival in Resectable Gastroesophageal Junction and Gastric Adenocarcinoma. Annals of surgery 263, 292-297, doi:10.1097/sla.0000000000001189 (2016). 
18. Grenader, T. et al. Prognostic value of neutrophil-to-lymphocyte ratio in advanced oesophago-gastric cancer: exploratory analysis of the REAL-2 trial. Annals of oncology: official journal of the European Society for Medical Oncology 27, 687-692, doi:10.1093/annonc/mdw012 (2016).

19. Takahashi, Y. et al. Neutrophil-Lymphocyte Ratio as a Prognostic Marker for Lung Adenocarcinoma After Complete Resection. World journal of surgery 40, 365-372, doi:10.1007/s00268-015-3275-2 (2016).

20. Junttila, M. R. \& de Sauvage, F. J. Influence of tumour micro-environment heterogeneity on therapeutic response. Nature 501, 346-354, doi:10.1038/nature12626 (2013).

21. Oh, S. Y., Kim, Y. B. \& Suh, K. W. Prognostic significance of systemic inflammatory response in stage II colorectal cancer. The Journal of surgical research 208, 158-165, doi:10.1016/j.jss.2016.08.100 (2017).

22. Sharma, D., Brummel-Ziedins, K. E., Bouchard, B. A. \& Holmes, C. E. Platelets in tumor progression: a host factor that offers multiple potential targets in the treatment of cancer. Journal of cellular physiology 229, 1005-1015, doi:10.1002/jcp.24539 (2014).

23. Lee, S. J. et al. Usefulness of neutrophil to lymphocyte ratio in patients with chronic obstructive pulmonary disease: a prospective observational study. The Korean journal of internal medicine 31, 891-898, doi:10.3904/kjim.2015.084 (2016).

24. Karataş, M., Gündüzöz, M., Öziş, T. N., Özakıncı, O. G. \& Ergün, D. Neutrophil to lymphocyte ratio and platelet to lymphocyte ratio as haematological indices of inflammatory response in ceramic workers' silicosis. The clinical respiratory journal 13, 159-165, doi:10.1111/crj.12997 (2019).

25. Tervaert, J. W., Stegeman, C. A. \& Kallenberg, C. G. Silicon exposure and vasculitis. Current opinion in rheumatology 10, 12-17, doi:10.1097/00002281-199801000-00003 (1998).

26. Cummings, M. et al. Preoperative neutrophil:lymphocyte and platelet:lymphocyte ratios predict endometrial cancer survival. British journal of cancer 113, 311-320, doi:10.1038/bjc.2015.200 (2015).

27. Mandaliya, H., Jones, M., Oldmeadow, C. \& Nordman, II. Prognostic biomarkers in stage IV non-small cell lung cancer (NSCLC): neutrophil to lymphocyte ratio (NLR), lymphocyte to monocyte ratio (LMR), platelet to lymphocyte ratio (PLR) and advanced lung cancer inflammation index (ALI). Translational lung cancer research 8, 886-894, doi:10.21037/tlcr.2019.11.16 (2019).

28. Mano, R. et al. The predictive role of preoperative and postoperative neutrophil-lymphocyte ratio in sarcomatoid renal cell carcinoma. Urologic oncology 37, 916-923, doi:10.1016/j.urolonc.2019.09.006 (2019).

29. Diem, S. et al. Neutrophil-to-Lymphocyte ratio (NLR) and Platelet-to-Lymphocyte ratio (PLR) as prognostic markers in patients with non-small cell lung cancer (NSCLC) treated with nivolumab. Lung cancer (Amsterdam, Netherlands) 111, 176-181, doi:10.1016/j.lungcan.2017.07.024 (2017).

30. Suh, K. J. et al. Post-treatment neutrophil-to-lymphocyte ratio at week 6 is prognostic in patients with advanced non-small cell lung cancers treated with anti-PD-1 antibody. Cancer immunology, immunotherapy: Cll 67, 459-470, doi:10.1007/s00262-017-2092-x (2018). 
31. Chen, Y. et al. Association of Dynamic Changes in Peripheral Blood Indexes With Response to PD-1 Inhibitor-Based Combination Therapy and Survival Among Patients With Advanced Non-Small Cell Lung Cancer. Frontiers in immunology 12, 672271, doi:10.3389/fimmu.2021.672271 (2021).

32. Lin, J. X. et al. Dynamic Changes in Pre- and Postoperative Levels of Inflammatory Markers and Their Effects on the Prognosis of Patients with Gastric Cancer. Journal of gastrointestinal surgery: official journal of the Society for Surgery of the Alimentary Tract 25, 387-396, doi:10.1007/s11605-02004523-8 (2021).

33. Kim, J. Y. et al. Dynamic changes of neutrophil-to-lymphocyte ratio and platelet-to-lymphocyte ratio predicts breast cancer prognosis. BMC cancer 20, 1206, doi:10.1186/s12885-020-07700-9 (2020).

34. Mason, M. et al. Neutrophil-lymphocyte ratio dynamics during concurrent chemo-radiotherapy for glioblastoma is an independent predictor for overall survival. Journal of neuro-oncology 132, 463471, doi:10.1007/s11060-017-2395-y (2017).

35. Gu, X. et al. Prognostic value of platelet to lymphocyte ratio in non-small cell lung cancer: evidence from 3,430 patients. Scientific reports 6, 23893, doi:10.1038/srep23893 (2016).

\section{Figures}


a
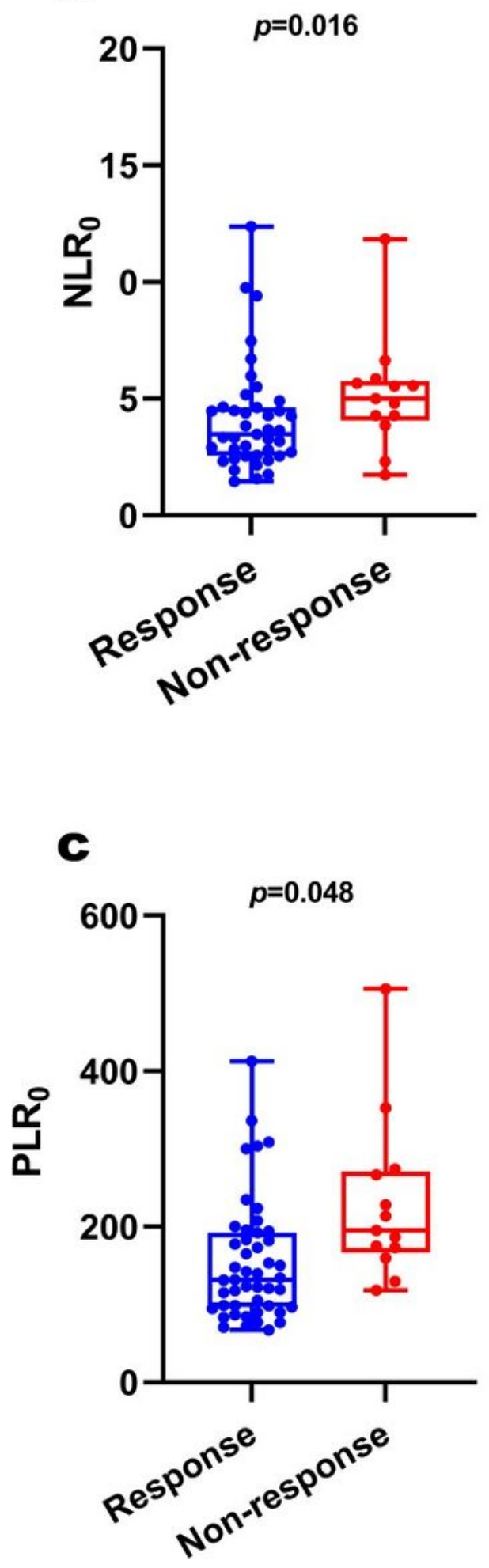

b
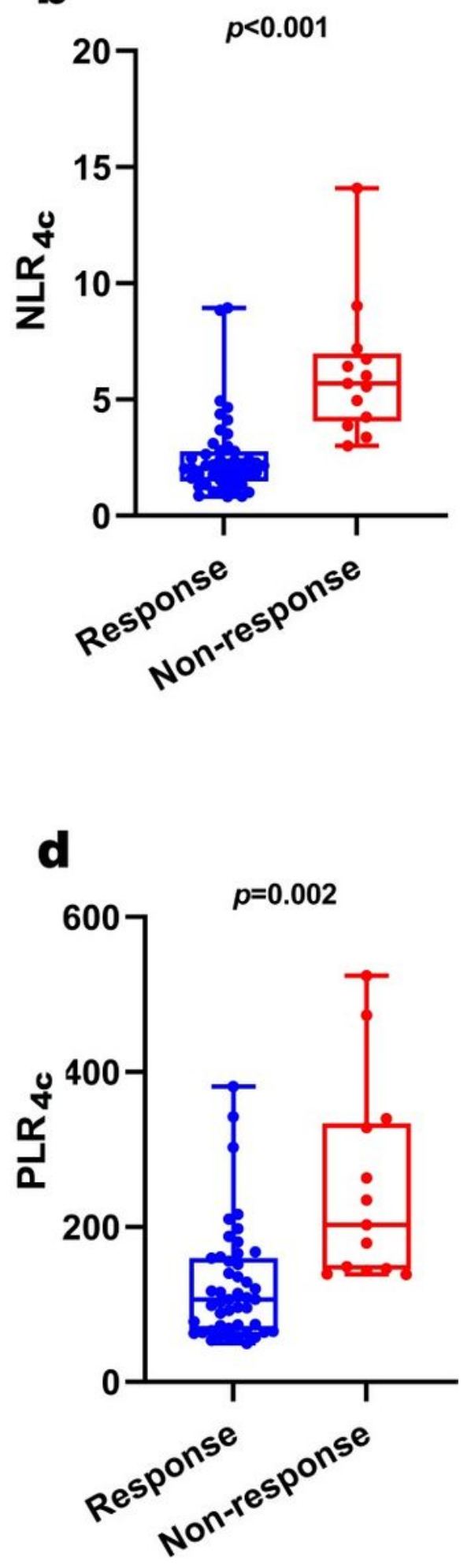

Figure 1

The distribution of NLR and PLR in the response and non-response group. a The distribution of NLRO in two groups. $\mathbf{b}$ The distribution of NLR4c in two groups. $\mathbf{c}$ The distribution of PLRO in two groups. $\mathbf{d}$ The distribution of PLR4c in two groups 

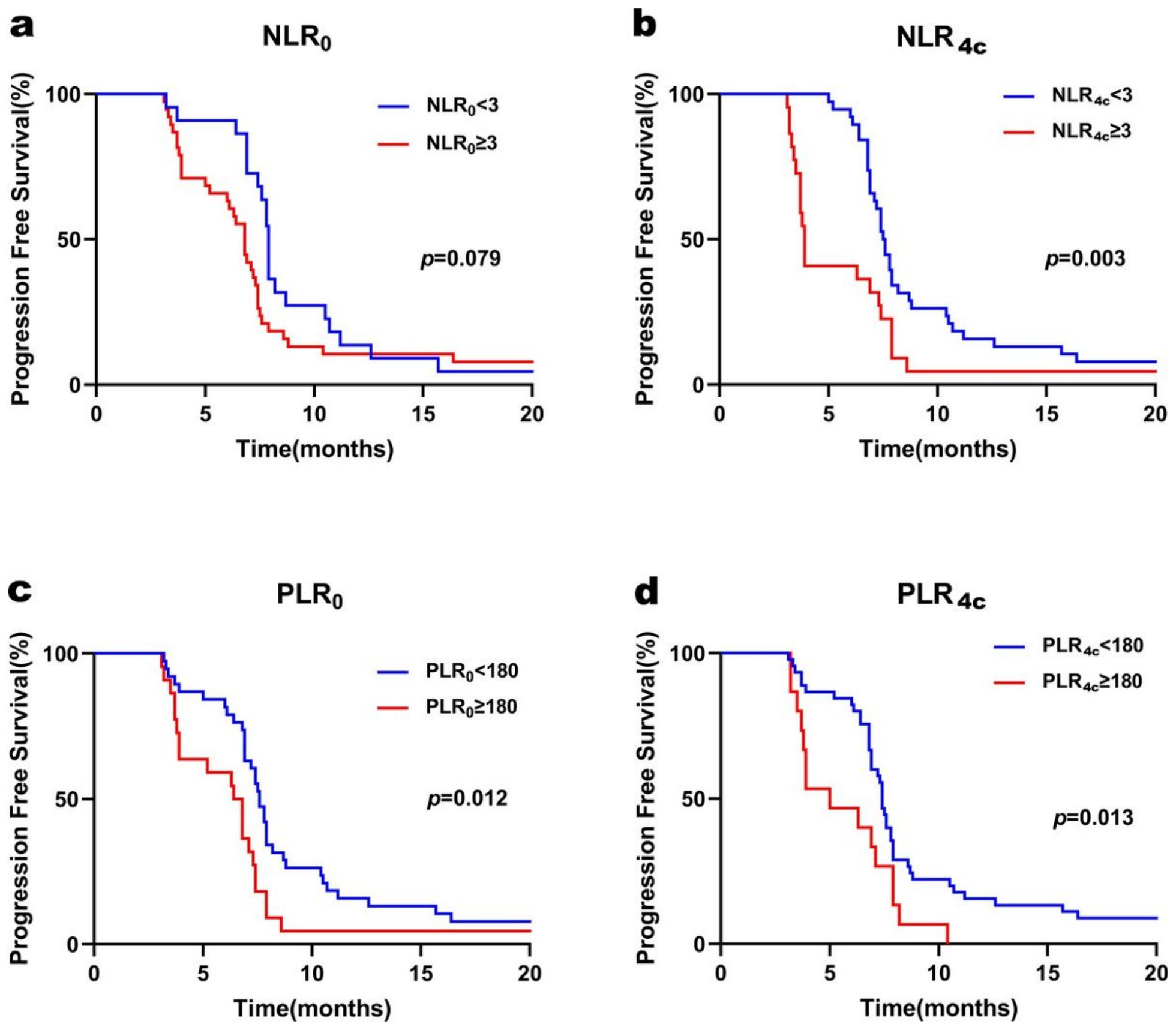

Figure 2

Kaplan-Meier curves for PFS according to NLRO (a), NLR4c (b), PLRO (c) and PLR4c (d) 
a

$\mathrm{NLR}_{\mathbf{0}}$

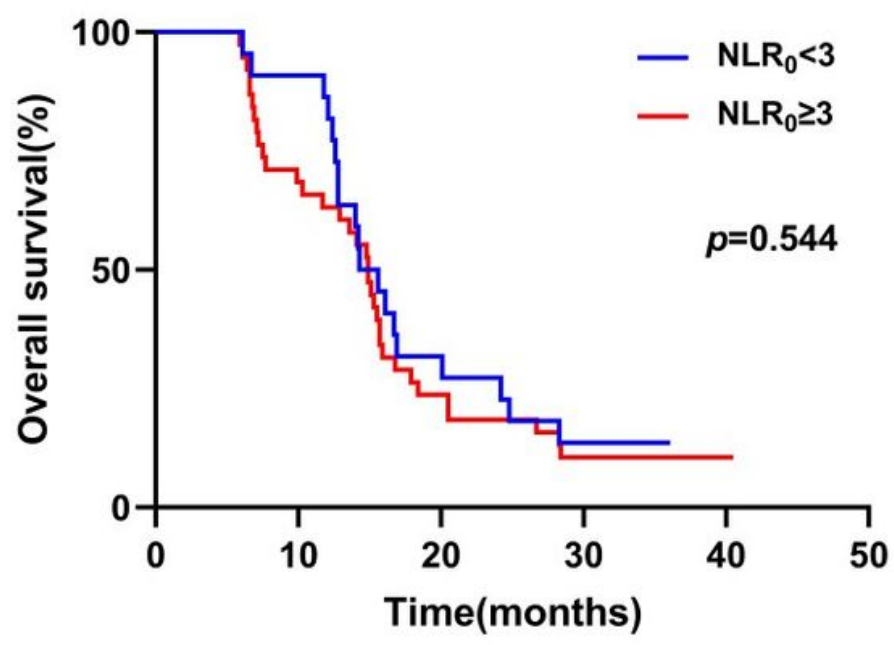

C

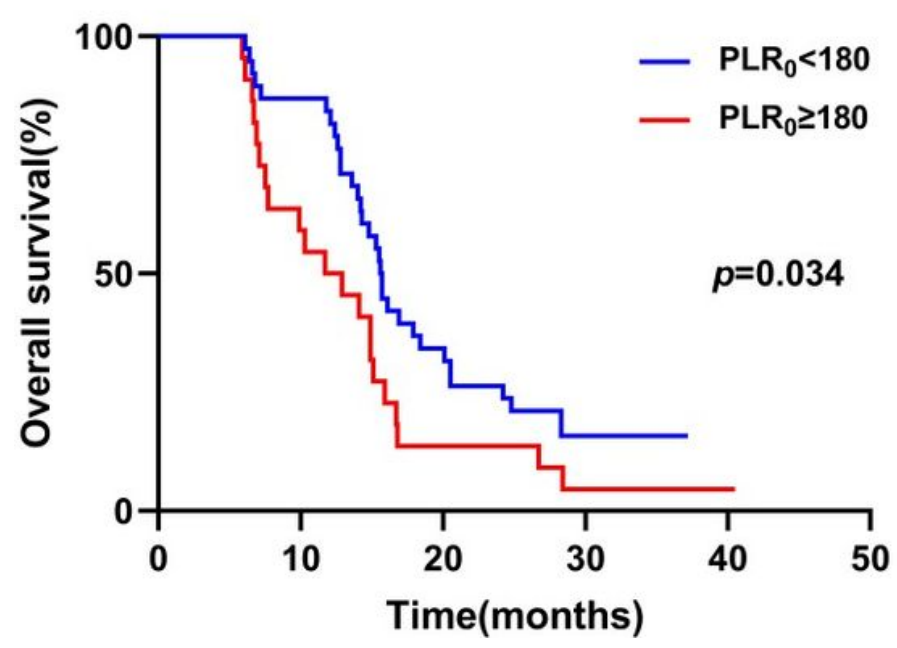

b

$\mathrm{NLR}_{\mathbf{4 c}}$

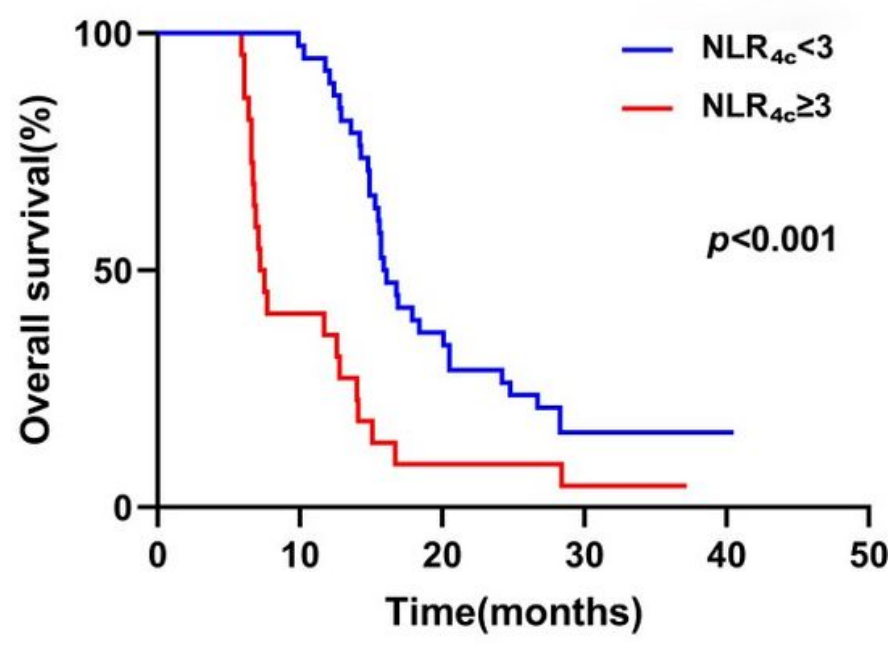

PLR $_{4 c}$

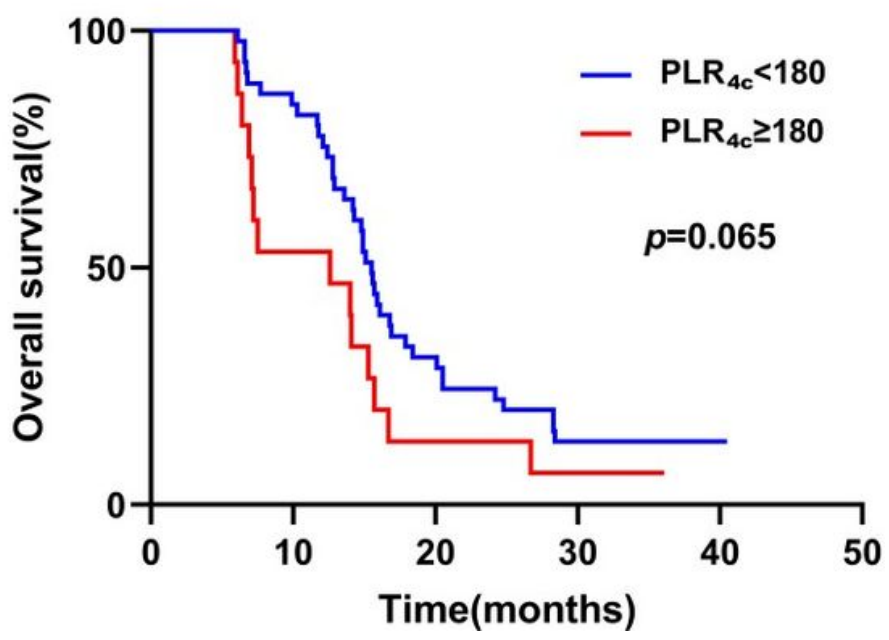

Figure 3

Kaplan-Meier curves for OS according to NLR0 (a), NLR4c (b), PLRO (c) and PLR4c (d) 
ปे

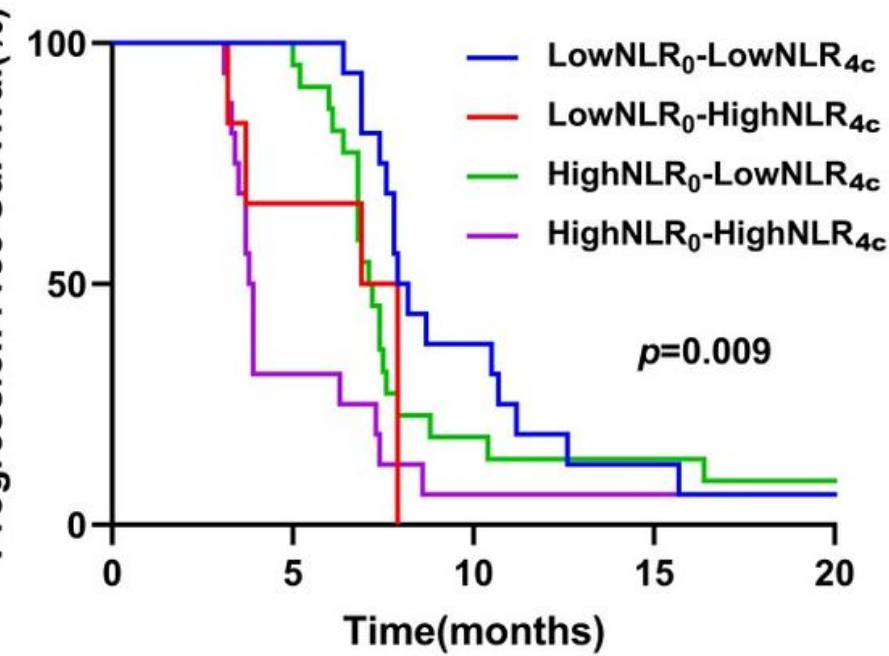

C

Changes of NLR

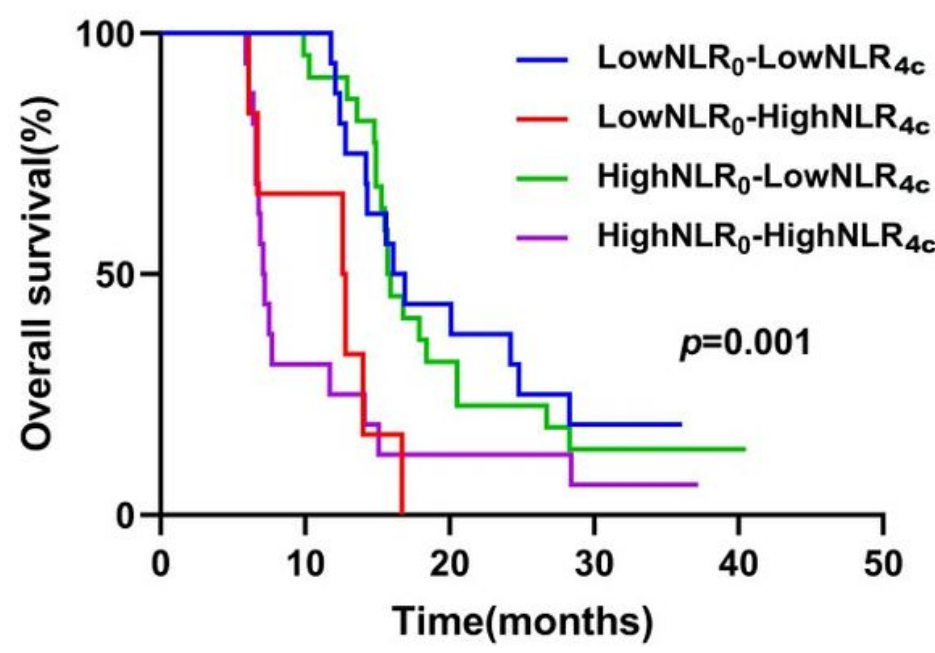

b Changes of PLR

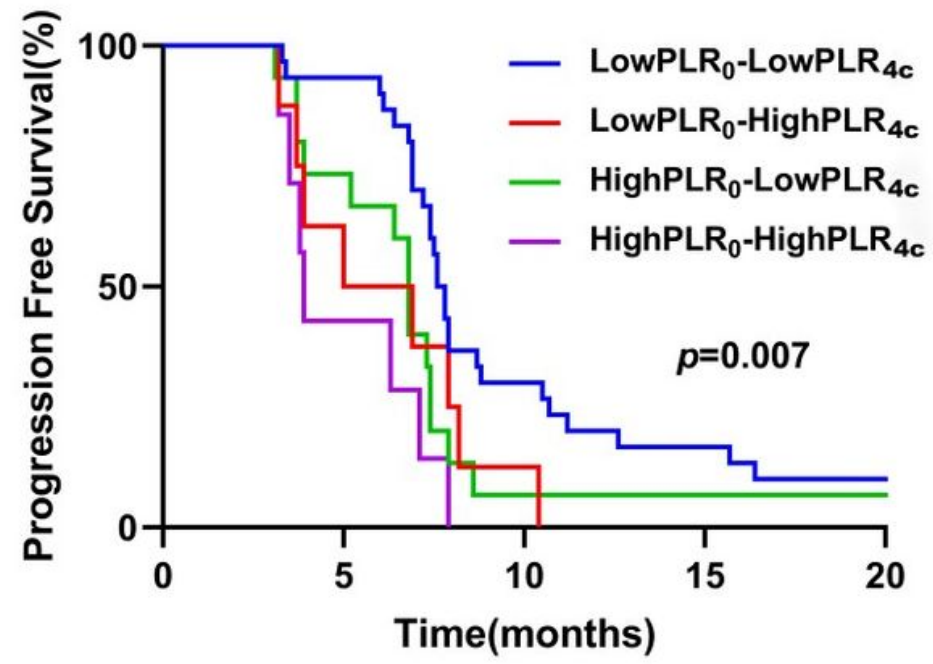

Changes of PLR

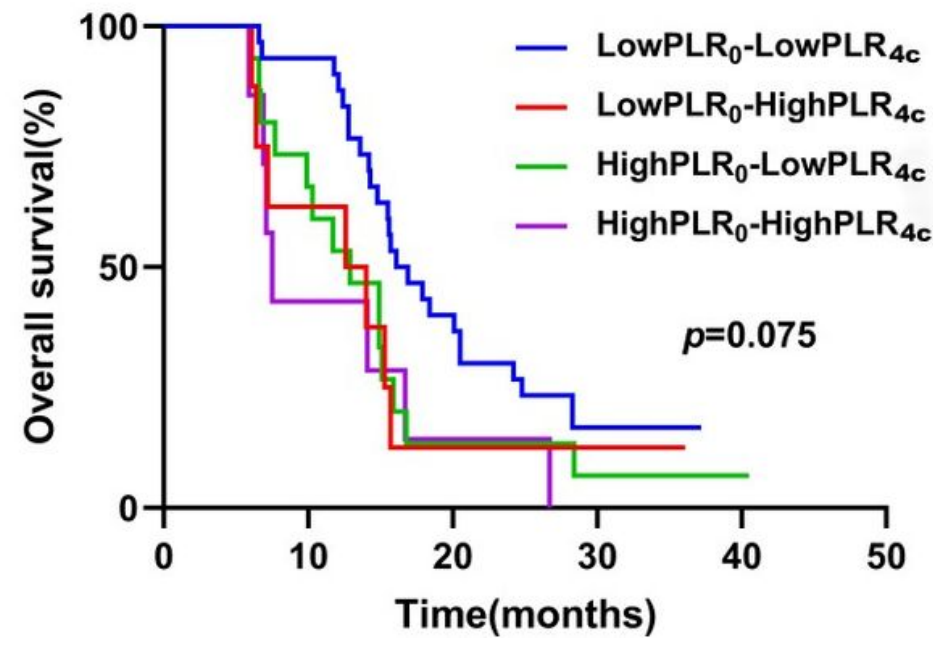

Figure 4

Kaplan-Meier curves for PFS and OS according to dynamic changes of NLR and PLR. a Dynamic changes of NLR for PFS. $\mathbf{b}$ Dynamic changes of PLR for PFS. $\mathbf{c}$ Dynamic changes of NLR for OS. $\mathbf{d}$ Dynamic changes of PLR for OS

\section{Supplementary Files}

This is a list of supplementary files associated with this preprint. Click to download.

- SupplementaryMaterials.docx 